\title{
Justin Beaumont, Christopher Baker (éd.), Postsecular Cities. Space, theory and Practice
}

London, Continuum, 2011, 276 p.

\section{Frédéric Dejean}

\section{(2) OpenEdition}

\section{Journals}

Édition électronique

URL : http://journals.openedition.org/assr/23436

DOI : $10.4000 /$ assr.23436

ISSN : $1777-5825$

Éditeur

Éditions de l'EHESS

Édition imprimée

Date de publication : 31 décembre 2011

Pagination : 104

ISBN : 9782713223273

ISSN : 0335-5985

Référence électronique

Frédéric Dejean, « Justin Beaumont, Christopher Baker (éd.), Postsecular Cities. Space, theory and

Practice », Archives de sciences sociales des religions [En ligne], 156 | octobre-décembre 2011, document 156-14, mis en ligne le 14 février 2012, consulté le 21 septembre 2020. URL : http:// journals.openedition.org/assr/23436; DOI : https://doi.org/10.4000/assr.23436

Ce document a été généré automatiquement le 21 septembre 2020.

(c) Archives de sciences sociales des religions 


\section{Justin Beaumont, Christopher Baker (éd.), Postsecular Cities. Space, theory and Practice}

London, Continuum, 2011, 276 p.

Frédéric Dejean

\section{RÉFÉRENCE}

Justin Beaumont, Christopher Baker (éd.), Postsecular Cities. Space, theory and

Practice, London, Continuum, 2011, 276 p.

1 L'ouvrage coordonné par Justin Beaumont (assistant de recherches à la Faculté des Sciences Spatiales de l'Université de Groningen) et Christopher Baker (directeur de recherche à la William Temple Foundation et maître de conférences en théologie publique et urbaine à l'Université de Chester) possède une double ambition : mettre l'espace, et plus précisément l'espace urbain, au cœur des études du fait religieux contemporain, d'une part, et prendre acte des conséquences du passage d'une société séculière à une société postséculière, d'autre part. L'approche par l'espace traduit la volonté d'interroger les nouvelles modalités du religieux dans les villes occidentales, qualifiées de «postmetropolis " pour reprendre l'expression du géographe américain Edward Soja. Notons cependant que l'analyse des formes spécifiques du religieux en contexte urbain ne constitue pas en soi une nouveauté, et les géographes ne sauraient avoir l'exclusivité d'une approche spatiale. Par exemple, les anthropologues insistent sur les configurations des lieux et les fonctions qu'ils assument dans les dynamiques communautaires locales (voir par exemple les contributions de Sandra Fancello et d'André Mary dans le collectif Lieux de sociabilité urbaine en Afrique sous la direction de Laurent Fourchard, Odile Goerg et Muriel Gomez-Perez).

2 Ici, l'originalité de l'entreprise tient avant tout dans le postulat selon lequel le religieux a changé de statut dans les sociétés contemporaines, et que ce changement serait tout 
particulièrement saillant en contexte urbain dans la mesure où la ville constitue désormais le cadre spatial de référence des sociétés occidentales. Il s'agit donc d'interroger ensemble les transformations urbaines les plus significatives et les formes nouvelles prises par le fait religieux au sein des sociétés. Les textes rassemblés montrent que loin de constituer le tombeau de la religion, la ville contemporaine est au contraire un formidable laboratoire dans lequel s'élaborent des pratiques religieuses nouvelles en phase avec le contexte socio-urbain.

3 Le titre de l'ouvrage éclaire le projet éditorial en renvoyant à deux auteurs sollicités par Beaumont et Baker. Il s'agit tout d'abord d'un hommage à La cité séculière : essai théologique sur la sécularisation et l'urbanisation de Harvey Cox, paru en 1965 dans son édition anglaise. Dans cet ouvrage, le théologien américain entendait conduire une réflexion associant les processus de sécularisation et d'urbanisation. Nous lisons : « Si la sécularisation désigne l'arrivée à l'âge adulte, l'urbanisation, elle, désigne le contexte de cet événement (p. 34) ». Ainsi, la ville constitue le contexte spatial de référence de la sécularisation. Mais la ville ne se définit pas uniquement de manière quantitative, par un nombre d'habitants (par exemple le seuil minimal de 2000 habitants en France) et une forte densité du bâti. La ville est avant tout une forme particulière d'organisation de la société : «L'urbanisation donne à la vie en commun une structure où la diversité tient la première place, avec le démantèlement des traditions (p. 34)». Soulignons au passage que la définition proposée par Cox est proche de celle formulée par Louis Wirth en 1938 dans un article fameux, "Urbanism as a way of life »: " For sociological purposes a city may be defined as a relatively large, dense, and permanent settlement of socially heterogeneous individuals (Wirth, 1938: 8)». S'inscrivant dans cette filiation, Postsecular cities entend rendre compte de ce nouveau contexte urbain dans lequel le fait religieux se déploie. La ville contemporaine n'est pas seulement un contexte passif, mais elle oriente les expressions spatiales et sociales de la religion.

4 Le titre renvoie également à plusieurs écrits de Jürgen Habermas publiés au cours des années 2000. Cette référence à l'héritier de l'École de Francfort est déterminante puisque Baker et Beaumont mobilisent dans leurs analyses ce qui fait le cœur du concept de postsécularité chez Habermas, à savoir un processus de renégociation de la place du religieux dans la sphère publique et dans le travail de résolutions de problèmes sociaux. Cette renégociation trouve un terreau particulièrement fertile dans le contexte des économies libérales où les organisations religieuses peuvent devenir des acteurs de choix face à des formes de désengagement public.

Pour autant, le recours au terme de " postséculier » ne va pas de soi. Dans une courte mais dense - préface, le géographe canadien David Ley s'interroge sur la pertinence d'introduire le terme de "postséculier ». Il souligne ainsi : "Could it be that what has changed is the focus of our gaze rather than the things themselves (p. xII)? » La question est d'autant plus pertinente que les sciences sociales des religions sont traversées par une ligne de fracture séparant les tenants de la «postmodernité» et ceux de $l^{\prime}$ « ultramodernité ». Alors que les premiers mettent en avant un changement de nature dans les formes d'expression et les rapports des faits religieux à la société globale, les seconds discernent davantage un changement de degré, nous serions ainsi dans une époque où les caractéristiques de la modernité se trouvent portées à leur paroxysme.

6 Dans l'introduction ("Introduction: the rise of the postsecular city »), le chapitre 2 («Postcolonialism and religion: new spaces of belonging and becoming in the postsecular city ») et la conclusion (« Afterword: postsecular cities »), Justin Beaumont 
et Christopher Baker font l'hypothèse d'un changement de nature, autant dans les modes d'insertion des religions dans le débat public que dans ses formes prises dans l'espace urbain. Ces trois textes méritent d'être lus ensemble, car ils constituent le cœur théorique du livre autour duquel les différentes contributions se déploient. Beaumont et Barker partent du constat que : «In this century, by contrast, religion, faith, communities and spiritual values have returned to the centre of public life, especially public policy, governance, and social identity (p.1)». Si l'espace privilégié par l'analyse est la ville c'est parce que les transformations pointées par les différentes contributions s'y expriment tout particulièrement. La proximité géographique de groupes religieux différents et leurs modes d'insertion différenciés dans l'espace urbain jouent un rôle essentiel dans l'émergence d'une société postséculière. Les deux auteurs distinguent sept lieux de débats abordés dans l'ouvrage: le retour de la notion de "sacré » dans le discours des aménageurs (1), l'urbain comme catégorie d'espace privilégiée dans les nouvelles formes de relations entre le religieux et le séculier (2), une volonté de faire consensus entre le discours religieux et le discours séculier dans la recherche du bien commun (3), la réactivation d'organisations religieuses dans le travail de justice social (4), le lien entre la croissance des Églises pentecôtistes et l'affirmation de l'économie néolibérale (5), de nouvelles formes de négociations entre le religieux et le politique, notamment dans le domaine des services publics (6), enfin les discussions entourant le multiculturalisme et ses implications dans le champ religieux (7).

7 Les quatorze contributions sont organisées en quatre parties de taille inégale: la première, "Mapping the theoritical terrains" ne contient qu'un seul texte («Postsecular cities and radical critique: a philosophical sea change?»). Les parties suivantes («Competing experiences of postsecular cities», "Postsecular policies and praxis » et "Theological and secular interpretations») offrent des analyses provenant de disciplines variées et explorent les différentes facettes de ces «villes postséculières ", à défaut de pouvoir en donner une définition ultime. Sans entrer dans le détail de chacun des textes, nous pouvons au moins souligner que les contributions les plus stimulantes sont celles qui font dialoguer approches théoriques et empiriques, et font l'effort de se confronter à la notion de "ville postséculière " pour voir comment elle réagit à l'épreuve des faits. Par exemple, le texte de Nynke de Witte, « exploring the postsecular state: the case of Amsterdam » (p. 203-222) se révèle passionnant. L'auteure montre comment les transformations des relations entre l'État hollandais et les groupes religieux se sont traduites sur le plan municipal, à Amsterdam, par des formes de coopérations nouvelles entre les communautés religieuses locales et les autorités publiques.

8 En revanche, certains textes ne se confrontent pas suffisamment à la notion de "cité post-séculière ", se contentent d'en faire vaguement référence, et ne montrent pas en quoi elle permet de renouveler l'approche de la religion dans les espaces urbains. Par exemple, la contribution de Greg Ashworth ne renvoie quasiment jamais à la "postsecular city» (si ce n'est dans le titre et dans l'avant-dernière phrase de la conclusion), de sorte que son texte - pourtant riche - aurait pu être inséré dans n'importe quel ouvrage traitant du religieux dans les sociétés contemporaines. Nous touchons là une des limites de l'ouvrage, d'ailleurs assez commune quand il s'agit d'entreprises collectives. 
9 Au final, Postsecular cities ouvre des perspectives stimulantes et invite les sciences sociales des religions à réfléchir aux traductions spatiales des nouvelles formes de participation des organisations religieuses dans le débat public. Néanmoins, l'ouvrage témoigne d'une difficulté à proposer une définition pratique de ce que peut être la " cité postséculière ». Cette difficulté tient sans doute davantage au caractère innovant - et donc aussi un peu tâtonnant - de la démarche qu'à un manque de fécondité de la proposition théorique de départ. 